\title{
APE: a breadboard to evaluate new phasing technologies for a future European Giant Optical Telescope
}

\author{
Frederic Gonte ${ }^{a}$, Natalia Yaitskova ${ }^{a}$, Philippe Dierickx ${ }^{a}$, Robert Karban $^{a}$ Alain Courteville ${ }^{b}$, \\ Achim Schumacher $^{c}$, Nicholas Devaney ${ }^{c}$, Simone Esposito ${ }^{d}$, Kjetil Dohlen ${ }^{e}$, Marc Ferrari ${ }^{e}$, \\ Luzma Montoya ${ }^{e}$ \\ ${ }^{a}$ European Southern Observatory,Karl-Schwarzschild-Str. 2, Garching, Germany; \\ ${ }^{b}$ Fogale Nanotech,285 rue Gilles Roberval, Nimes, France; \\ ${ }^{c}$ Instituto de Astrofisica de Canarias, Address,La Laguna, Tenerife,Espana; \\ ${ }^{d}$ Arcetri Astrophysical Observatory,Largo E. Fermi, Florence, Italy; \\ ${ }^{e}$ Laboratoire d'Astrophysique de Marseille,2 Place Leverrier, Marseille, France
}

\begin{abstract}
The point spread function of a segmented aperture is seriously affected by the misalignment of the segments. Stringent requirements apply to position sensors and their calibration. The Active Phasing Experiment (APE) will be a technical instrument aimed at testing possible phasing techniques for a European Giant Optical Telescope (EGOT) in a representative environment. It will also integrate simultaneous control of segmented and monolithic, active surfaces. A mirror composed of 61 hexagonal segments is conjugated to the primary mirror of the VLT. Each segment can be moved in piston, tip and tilt and can be controlled in open or closed loop. Three new types of Phasing Wave Front Sensors dedicated to the measurement of segmentation errors will be tested, evaluated and compared: a modified Mach-Zehnder sensor developed by the LAM and ESO, a Pyramid Sensor developed by Arcetri, and a Curvature Sensor developed by IAC. A reference metrology developed by FOGALE will be added to measure directly the deformation of the segmented mirror and check the efficiency of the tested wavefront sensors. This metrology will be based on a synthetic wavelength instantaneous phase stepping method. This experiment will first run in the laboratory with point-like polychromatic sources and a turbulence generator. In a second step, it will be mounted at a Nasmyth focus of a VLT unit telescope. These activities are included in a proposal to the European Commission for funding within Framework Program 6.
\end{abstract}

Keywords: giant telescope, segmented mirror, phasing wavefront sensor, active optics

\section{INTRODUCTION}

The essential purpose of the APE experiment is to explore, integrate, and validate active, that is low temporal frequency, wavefront control schemes and technologies for an EGOT. This includes the evaluation and comparison of the performance of different types of wavefront sensors in the laboratory and on the sky on the one hand and the integration of the control of a segmented aperture control into an already existing active system (including field stabilization and active optics) and driving both the active system and the control of the segments from the output of the full system on the other hand.

To accomplish these taks APE will be designed as a technical prototype which will be installed and tested at a Nasmyth focus of a VLT unit telescope. The telescope provides all active functions (field stabilization, focusing, centering, active deformable mirrors) and the APE instrument emulates the optical effects of segmentation. The latter is done within APE by reimaging the telescope pupil onto a small Active Segmented Mirror (ASM) whose shape is measured by an internal metrology (IM). The ASM is composed of 61 hexagonal segments and has a diameter of approximately $15 \mathrm{~cm}$. Each segments is controlled in piston, tip and tilt.

The final wavefront is measured by three new types of Phasing WaveFront Sensors (PWFSs), combined in the Phasing Metrology Module. The new types of PWFSs are a modified Mach-Zehnder interferometer, a curvature

\footnotetext{
Further author information: (Send correspondence to Frederic Gonte.)

E-mail: fgonte@eso.org, Telephone: 00498932006852
} 


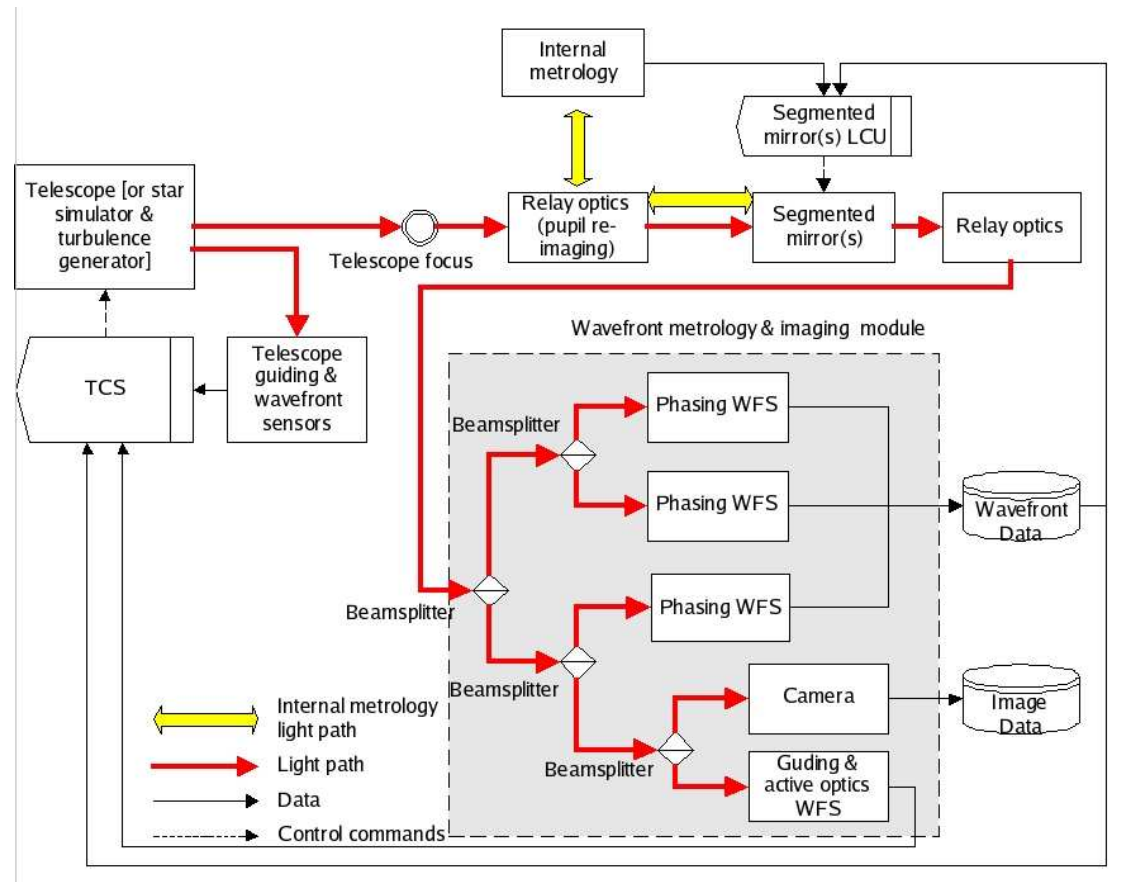

Figure 1. APE Schematic

sensor and a Pyramid sensor. For reference and comparison APE will also be equipped with a Shack-Hartmann sensor capable of measuring phase errors at segment edges.

APE is a four years project which starts in July 2004.

\section{APE PRINCIPLE}

Fig. 1 shows the principal components of APE. The light of the star collected by a VLT unit telescope or generated by a reference source and a turbulence generator is captured by the relay optics which re-images the primary mirror onto the ASM described in Ch. 3.4. The reflected light is then distributed to the different phasing sensors (see Ch. 4) and an imaging camera (see Ch. 3.3). An Internal Metrology (IM) described in Ch. 3.5 will measure the exact positions of the each of the 61 segments. It provides an independent check of the corrections applied to the segmented mirror. The ASM is controlled by the Telescope Control System (TCS). APE can work either be used to compare the measurements of the various phasing sensors with the measurements done by the internal metrology or work as a closed-loop control system correcting the telescope aberrations and the misalignments of the segmented mirror based on the measurements from one or more of the phasing sensors. Thereby it also serves as a testbed for the development of a control system for active optics of a telescope with segmented and flexible mirrors.

\section{APE SUB-ELEMENTS}

This chapter describes all components of APE except the Phasing sensors which are introduced in chapter 4). Fig. 2 shows the main components and elements of APE. In the hardware there is a clear distinction between the new PWFSs and the Shack-Hartman sensor (called Active Optics WFS), which is considered part of the Guiding \& active optics group. It will be a reference against which the other sensors are tested. It will also supply information on the deformations of the meniscus mirrors in case this can not be delivered by the PWFS. In the Segmented mirrors group there is one in-pupil mirror, the ASM, and one out of the pupil mirror which will simulate a secondary segmented mirror on a giant telescope. The latter will either be a static segmented mirror or a transmissive phase plate. 


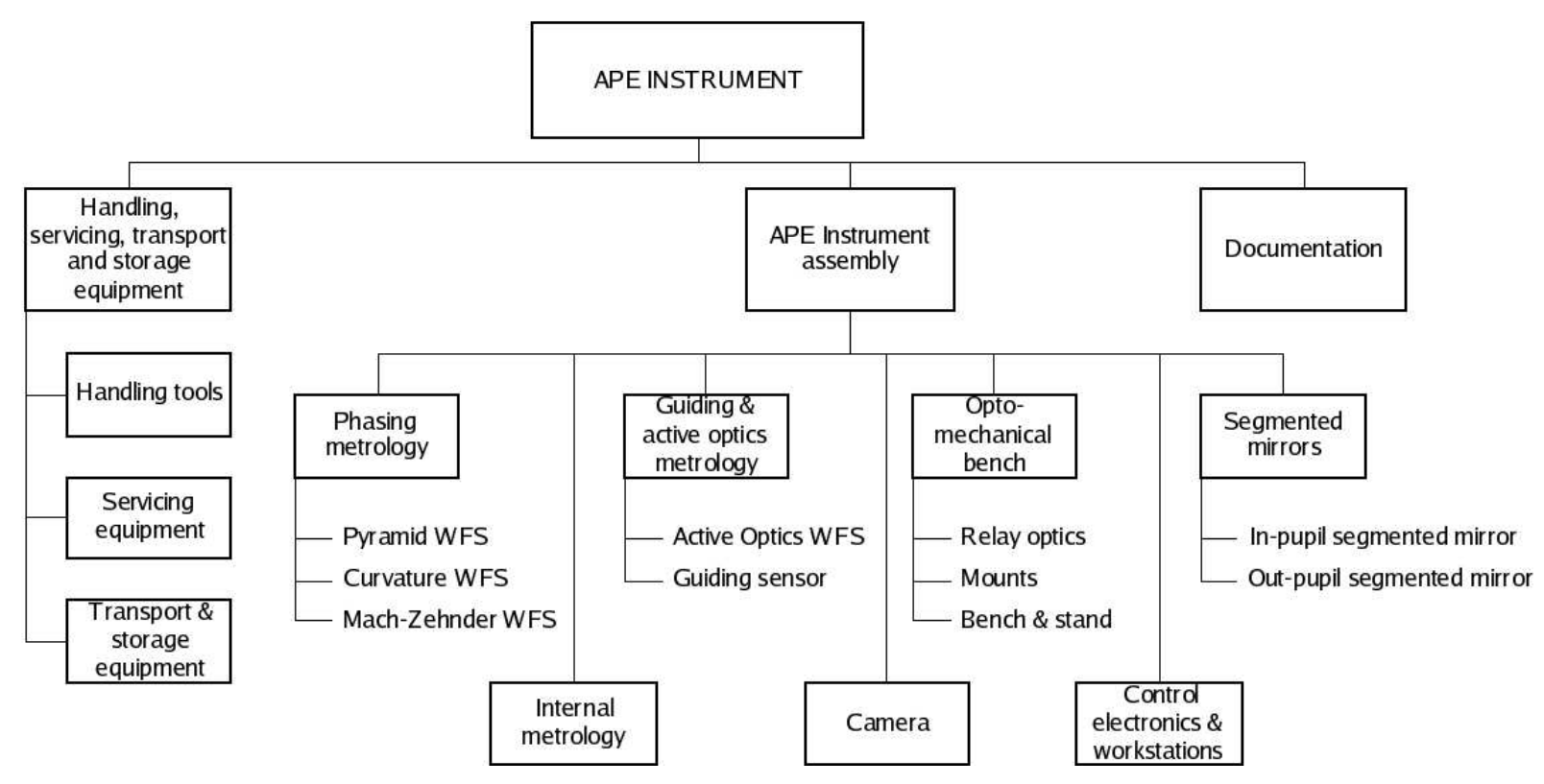

Figure 2. APE hardware Tree

\subsection{Optical set up}

Fig. 3 shows the optical design. The optical beam at the Nasmyth focus with a focal ratio of F/15, is collimated by a collimator, reflected by the segmented mirror, and then refocused by another collimator with the same focal ratio as the first collimator. The beam is then split into 5 different beams for the Phasing WFSs and the imaging camera. A system of two tip-tilt mirrors directs the light of a star or of the reference source to the WFSs. Each PWFS receive the same intensity. The static out of pupil mirror or piston plate is not represented in this design.

\subsection{Mechanical set up}

APE shall be mounted on an optical table with a size of 1.5 by $2.5 \mathrm{~m}$. Since APE will be installed on the Nasmyth platform of a VLT unit telescope all the all components have to be compliant with the VLT standard. The derotator will directly be mounted on the interface of the VLT Nasmyth platform. The stability of the star on the phasing wavefront sensor shall be better than 0.1 arcsec. The calibration system is a copy of the MAD calibration system (see Marchetti \& al. ${ }^{1}$ ).

\subsection{Imaging camera}

The imaging camera is composed of an ESO technical CCD having $1024 * 1024$ pixels with a size of $13 * 13$ microns. The CCD is Peltier cooled to less than -35 degrees Celsius and has a typical readout noise of less than below $50 \mathrm{e} / \mathrm{pixel} / \mathrm{sec}$. Its quantum efficiency is better than to $85 \%$. The camera shall have a resolution better than $0.1 \mathrm{arcsec} / \mathrm{pixel}$ and a field of view of 1 arcmin. Because of the focal ratio of F/15 at the VLT Nasmyth focus, which is equivalent to 582 microns/arcsec, we need additional optics to obtain a magnification of $1 / 2.7$.

\subsection{Active segmented mirror}

The segmented mirror is composed of 61 hexagonal segments as shown in Fig. 4. The size of the segments flat to flat is between 15 and $23 \mathrm{~mm}$. The gap between the segments is $0.15 \mathrm{~mm}$. The segments are aluminum coated to obtain a reflection of more than $85 \%$ from $450 \mathrm{~nm}$ to $1400 \mathrm{~nm}$. The misfigure of the segments shall be less than 30 $\mathrm{nm}$ wavefront RMS. The segments are controlled in piston, tip and tilt, and the range of the position actuators shall be between \pm 5 and \pm 10 microns. The segmented mirror shall have has a closed loop bandwidth of $5 \mathrm{~Hz}$. 


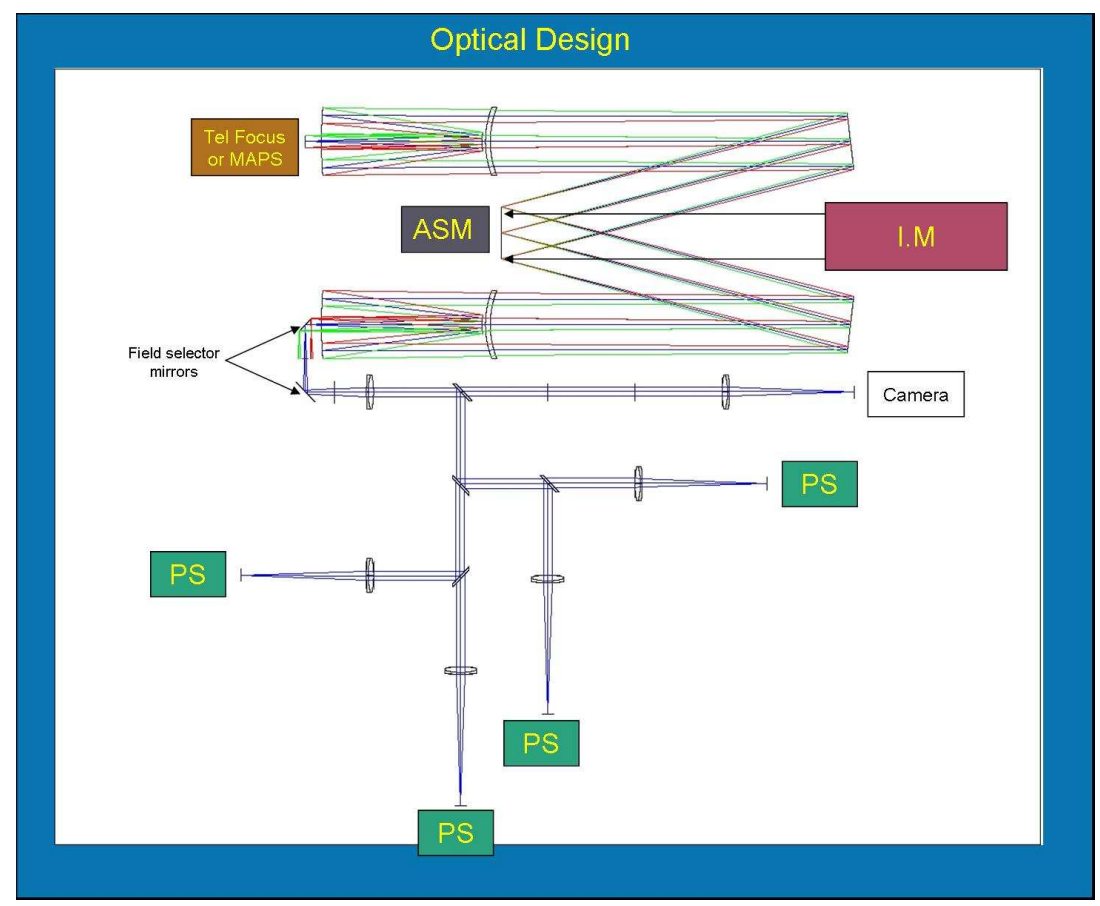

Figure 3. APE Optical Design

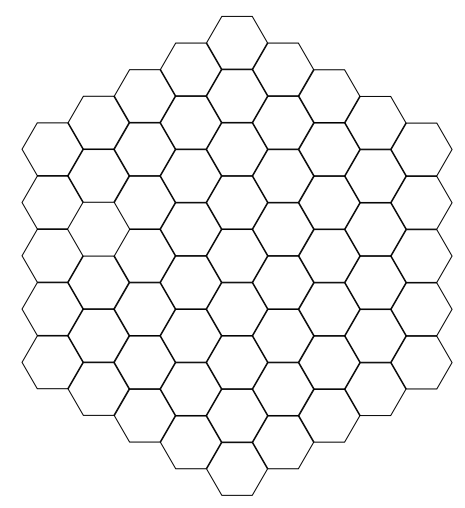

Figure 4. ASM design. 


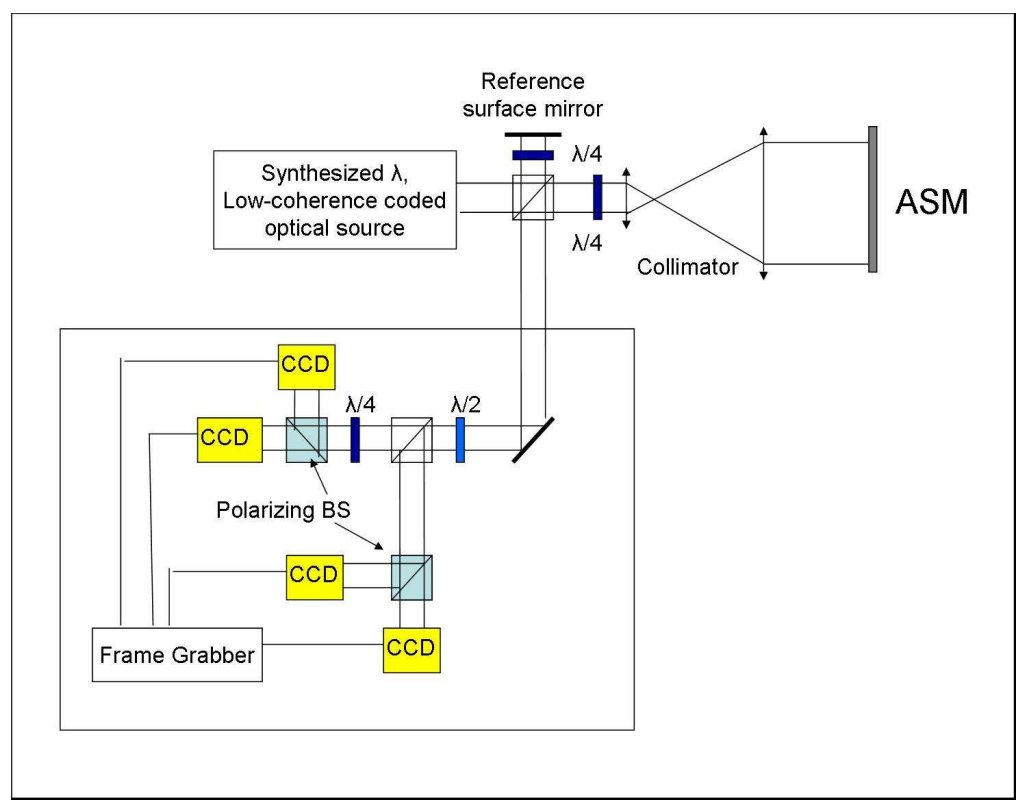

Figure 5. Internal Metrology design.

\subsection{Internal metrology}

The internal metrology is delivered by FOGALE nanotech. It is a synthetic wavelength interferometer. This is a polarization Twyman-Green interferometer illuminated by a synthesized wavelength low-coherence coded optical source. The synthetic wavelength is obtained from 2 wavelengths and can be adjusted by choosing the right separation between $\lambda_{1}$ and $\lambda_{2}$ and is given by

$$
\Lambda=\frac{\lambda_{1} \lambda_{2}}{\lambda_{1}-\lambda_{2}}
$$

The two wavelengths are $850 \mathrm{~nm}$ and $800 \mathrm{~nm}$ which gives a synthetic wavelength of 13.6 microns. The resolution of the piston measurement on the segments will be $1 \mathrm{~nm}$ RMS. Fig. 5 shows the proposed optical layout based on a Twyman-Green interferometer which measures the optical path difference between the reference mirror and the test surface (ASM).

\subsection{Turbulence Generator}

A combined star simulator and turbulence generator named MAPS has been developed, mounted and tested at ESO (See Kolb \& al. ${ }^{2}$ ). It can simulate the effects of three turbulent layers in the atmospheres at different altitudes with a total seeing of up to 0.65 arc second using three transmissive phase screens which are conjugated to the three altitudes. It also simulates a field of 2 arcminutes containing up to 34 stars. This turbulence generator will first be used in the experiment MAD (see Marchetti \& al. ${ }^{1}$ ) and then in APE.

\section{PHASING WAVEFRONT SENSORS}

One of the goals of APE is to compare simultaneously the performance of several PWFSs. They will therefore receive the same amount of light for simultaneous exposures and will use the same VLT technical CCDs with identical control systems. The four PWFSs to be compared are MAZES proposed by LAM, PYPS proposed by ARCETRI, DIPSS proposed by IAC/GTC, and a SHAPS which will be supplied by ESO. 


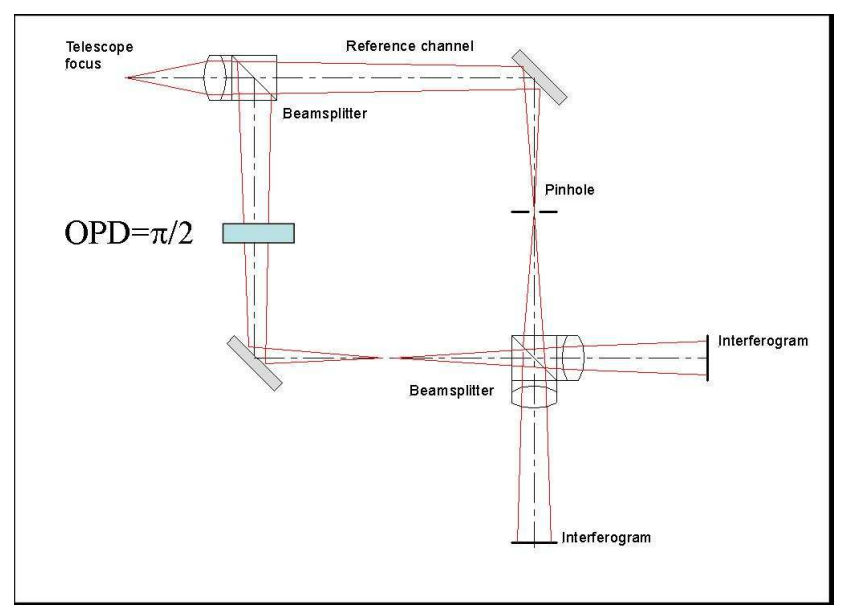

Figure 6. MAZES Principle.

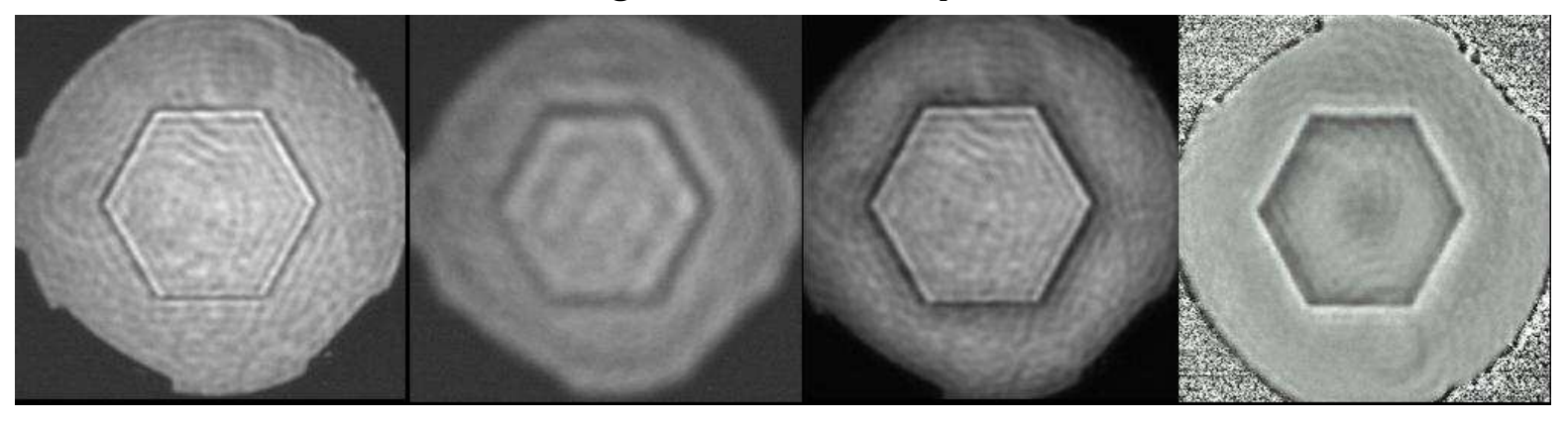

Figure 7. Measured intensity distribution of arm 1(a), arm 2(b), the interferogram(c) and of the normalized signal(d).

\subsection{MAZES: Modified Mach-Zehnder Phasing Sensor}

MAZES will be designed by LAM and ESO. A schematic view is given in Fig. 6. Its principle has been developed by Natalia Yaitskova, Kjetil Dohlen and Luzma Montoya (see Yaitskova \& al. ${ }^{3}$ ). The telescope beam is focused inside the Mach-Zehnder interferometer. In one of the two arms at the point spread function at the location of the focus will be filtered spatially by a pin hole with a size of the same order as the size of the image, that is the diffraction pattern in the case without and the seeing pattern in the case with atmosheric disturbances. The segmented mirror is then reimaged after the interference via a lens onto the detectors. The optical phase difference between the two arms must be equal to $\pi / 2$. This can be achieved by alignment or with a phase plate. The signal is obtained by taking the difference of intensities between the two arms. A prototype has been mounted at the Observatoire de Marseille and tested with a piston mirror. During the test a turbulence generator simulated seeing up to 0.45 arcsecond and $r_{0}=25.8 \mathrm{~cm}$ at $680 \mathrm{~nm}$. The recorded signals are shown in Fig. 7.

\subsection{PYPS: Pyramid Phasing Sensor}

The pyramid sensor has first been proposed by R. Ragazoni (see R. Ragazzoni ${ }^{4}$ ) for use in adaptive optics. PYPS will be developed by ARCETRI. A preliminary theoritical and experimental study has been made by S. Esposito (see Esposito \& al. ${ }^{5}$ ) to adapt it to the phasing of mirror segments. According to this study with a star of visual magnitude 15 the resolution should be better than $40 \mathrm{~nm}$ RMS. The principle of PYPS is shown in Fig. 8. 


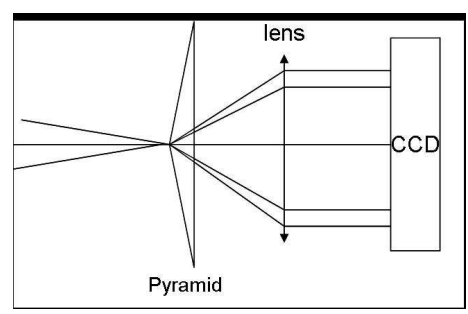

Figure 8. Principle of PYPS.

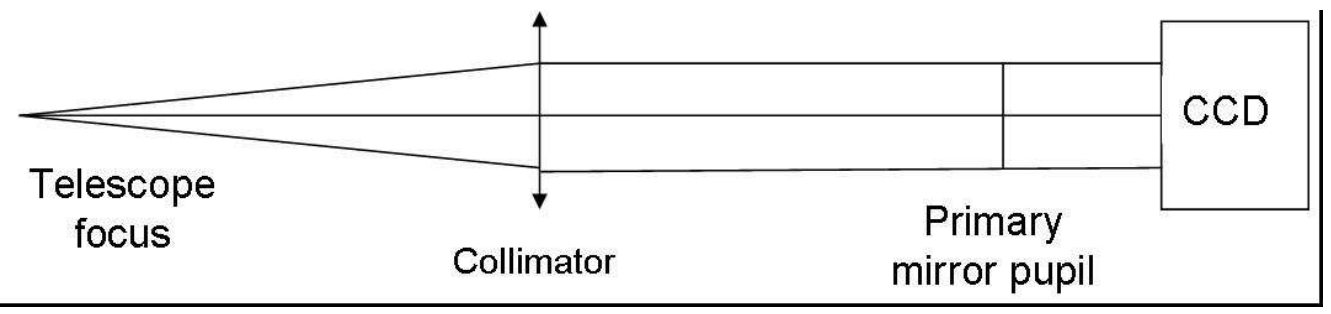

Figure 9. DIPSS Schematic.

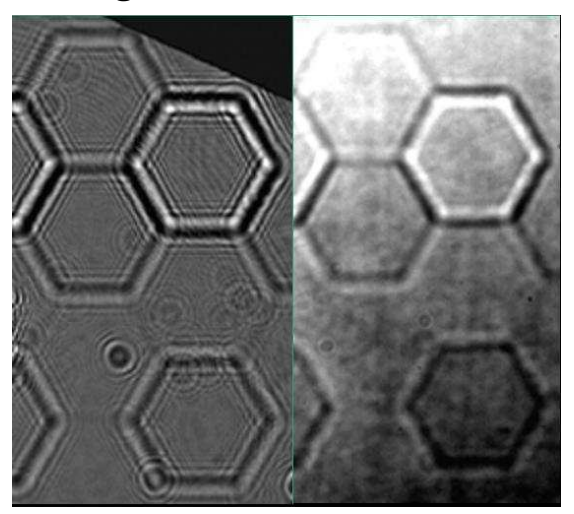

Figure 10. (a)DIPSS signal no turbulences (b) DIPSS signal with seeing $=0.65$ arcsec.

\subsection{DIPSS:Diffraction Image Phase Step Sensing}

The principle of a curvature sensor has been first described by Roddier. The Institute of Astrophysics of the Canaries Island (IAC) proposes a modified curvature sensor called DIPPS. The principle has been developed by Achim Schumacher. (see Schumacher \& al. ${ }^{6}$ ). A schematic view is shown in Fig. 9.

A preliminary experiment has been done in Garching using a turbulence generator and a piston plate, The piston plate was composed of 37 segments with 4 levels of piston steps and was placed on the pupil position of the primary mirror. The experiment has been done with and without turbulence equivalent to a seeing up to 0.6 arcsec. The first analysis of the result shows an error of less than $15 \mathrm{~nm}$ RMS without turbulences and better than $25 \mathrm{~nm}$ RMS with seeing of 0.6 arcsec. Further analysis will be done. Fig: 10 shows the signal without and with atmospheric turbulence.

\subsection{SHAPS: Shack-Hartmann Phasing Sensor}

The Shack-Hartmann sensor called SHAPS will be developed by ESO. It will be the reference for the other PWFSs and it will be used also as the guiding and active optics WFS. An ESO technical CCD will be used with 


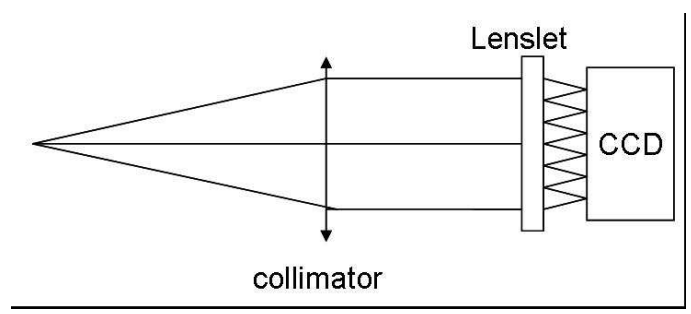

Figure 11. SHAPS.

a size of $1024 * 1024$ pixels. The microlenses will be designed to specifically measure piston, tip and tilt of each segment. Its principle is shown in Fig. 11.

\section{APE CONTROL SYSTEM}

The Active Phasing Experiment Control Software (APECS) is required to carry out the APE project. The control software has to provide the means to interface, control and monitor the various devices needed for this project. The software will provide all the necessary functionality to evaluate the control system under laboratory conditions and under the conditions in the VLT environment.

The system must accomplish the coordination between the wavefront analyses done by several PWFSs, control of the segment mirror ASM and the VLT active optics and the coordination between the imaging camera used to select the star and the field selector composed of 2 scanning mirrors. It shall control all TCCDs (Technical CCD) (from the PWFSs and of the imaging camera), the field selector and its scanning mirrors, the ASM, the 3 -axis table of the calibration unit, the derotator since APE will be on the Nasmyth platform, the Z-tables for the imaging camera and the PWFSs (focusing).

APECS shall deliver the control loops of the ASM in open and closed loop with the IM or with any of the PWFS at up to $5 \mathrm{~Hz}$.

The system shall provide extended test facilities, both for individual APE devices and for higher level operation. The system shall interface the IM with ASM control LCU (Local Control Unit), it also shall interface and control the VLT TCS-active optics of M1 and M2 with the data from the PWFS and active optics WFS.

The baseline of the control software will be the VLT Common Software (VLTSW).

\section{APE MEASUREMENTS CAMPAIGNS}

APE Technical runs shall last a year with a first period in laboratory and then a second period on the VLT. The first period shall last 6 months. the second is on the Paranal mountain and shall consist of 3 campaigns of the maximum duration of 2 weeks each. Between each campaign we shall reserve 2 to 3 months for the processing and analysis of the measurements and planning of the next campaign.

\section{REFERENCES}

1. E. Marchetti and al, "MAD status report," in Advancements in Adaptive Optics, Proc. SPIE 5490, 2004.

2. J. Kolb and al, "MAPS: a turbulence simulator for mcao," in Advancements in Adaptive Optics, Proc. SPIE 5490, 2004.

3. N. Yiatskova and al, "A Mach-Zehnder phasing sensor for extremely large segmented telescopes: Laboratory results and close loop algorithm," in Ground-based Telescopes, Proc. SPIE 5489, 2004.

4. R. Ragazzoni, "Pupil plane wavefront sensing with an oscillating prism," J. Mod. Opt. 43, pp. 289-293, 1996.

5. S. Esposito and al, "Co-phasing of segmented mirrors using pyramid sensor," in Astronomical Adaptive Optics Systems and Applications, R. Tyson, ed., Proc. SPIE 4839, pp. 72-78, 2003.

6. A. Schumacher and N. Devaney, "DIPSS: cophasing segmented mirrors with minimal hardware requirements," Applied Optics. To be published, 2004. 